Rev. Biol. Trop., 47(1-2): 225-236, 1999

www.ucr.ac.cr www.ots.ac.cr www.ots.duke.edu

\title{
Anidación de la tortuga Dermochelys coriacea (Testudines: Dermochelyidae) en playa Gandoca, Costa Rica (1990 a 1997)
}

\author{
Didiher Chacón Chaverri
}

Programa Marino/Humedales, Asociación ANAI, Apdo. 170-2070, Sabanilla, Montes de Oca, San José, Costa Rica, Fax: 506-224-3570, Internet: anaicr@ sol.racsa.co.cr.

Recibido 12-I-98. Corregido 7-IX-98. Aceptado 19-X-98.

\begin{abstract}
The nesting of the leatherback sea turtle was studied in Gandoca Beach, an important nesting beach on the southeastern Caribbean coast of Costa Rica, in the Gandoca/Manzanillo National Wildlife Refuge. Nesting was recorded from 1990 to 1997 (each February through July). Nesting peaked in April and May (depending on the year); 16 biological paramenters were recorded and part of the rookery was tagged with monel tags. During this period 1045 females were studied and 3484 nests were recorded; 534 nests was the yearly mean, (range 226-1 135), $20.8 \%$ of the nests were relocated to hatcheries, as a conservation effort to prevent loss of nests. Mean curve carapace length was $154.65 \mathrm{~cm}$ and width $112.83 \mathrm{~cm}$. Mean normal number of eggs/nest was 79.28 and 35 yolkless. Each turtle laid an average of 2.5 nests per season with an internesting interval of 9 days. In 1997, 39\% of the nesting females had been previously tagged in Gandoca Beach and a few also in Colombia (Urabá Gulf), Pacuare and Tortuguero (Costa Rica). Poaching activity, beach debris and extensive erosion represent the main hazards that are leading to a decline of the sea turtles in Gandoca.
\end{abstract}

Key words: Reptilia, Leatherback, Dermochelyidae, Dermochelys coriacea, nesting biology, sea turtle, tagging, remigration, poaching, conservation, Caribbean, Costa Rica.

En la actualidad existen ocho especies de tortugas marinas (Márquez 1990) y seis de ellas anidan en Costa Rica, Chelonia mydas (Linnaeus 1758), Eretmochelys imbricata (Linnaeus 1766), Dermochelys coriacea (Vandelli 1761), Lepidochelys olivacea (Eschscholtz 1829), Caretta caretta (Linnaeus 1758), Chelonia agassizii (Bocourt 1868); el país cuenta además con dos de las cinco playas donde se presentan arribadas de tortuga lora o carpintera en el mundo (Cornelius et al. 1986), la colonia más importante de tortugas verdes del mar Caribe (Carr et al. 1990); la playa más importante de anidación de tortugas baulas del Pacífico central (Williams et al. 1995), además de colonias muy importantes de tortugas carey en los ecosistemas de arrecifes de coral del mar Caribe (Meylan et al. 1997).

La tortuga baula está clasificada como una especie en peligro de extinción por la Unión
Internacional para la Naturaleza (UICN 1995); esta especie pasa su ciclo de vida mayormente en aguas oceánicas alimentándose de invertebrados como nidarios (Cyanea sp.) y tenóforos. (Leslie et al. 1996). La baula es la única especie de tortuga marina que puede habitar en aguas oceánicas con temperaturas extremas distribuyéndose desde zonas subpolares hasta los trópicos (Leslie et al. 1996) donde migra para desovar en importantes playas de anidación como en Guyana Francesa, Surinam, México y Costa Rica. Sin duda alguna los litorales de Costa Rica, son muy importantes para estos reptiles no sólo como ecosistemas para desovar sino como sitios de copulación y alimentación.

Numerosos estudios sobre marcaje, biología reproductiva y conservación de tortugas baulas se han desarrollado en la parte norte de la costa caribeña de Costa Rica (Hirth y Ogren 
1987, Campbell, Lagueux y Mortimer 1996, Leslie et al. 1996), pero muy poco se sabe del desove de esta especie en la zona sur del Caribe. Algunas de estas playas de anidación de tortugas marinas están en el Refugio Nacional de Vida Silvestre GandocalManzanillo (REGAMA) en Talamanca. Este incluye 4436 hectáreas de área marina, con dominancia de fondos duros (principalmente coral y arena) (Yesaki y Giudicelli 1971).

El papel que juegan las tortugas marinas en las economías locales y en varios casos regionales está supeditado a los bienes y servicios que ellas brindan a los pobladores de las costas; su explotación se realiza desde hace siglos como fuentes de carne, huevos, materia prima para cosméticos, joyería y artesanía (UICN 1995, Meylan et al. 1997); y en las últimas décadas como elementos de atracción para el turismo (Herzog y Gerrand 1992); estas condiciones las hacen recursos de inmensa importancia.

Sin embargo, a pesar de estas magnificativas cualidades estas especies se encuentran perjudicadas por diversos factores que han declinado sus poblaciones, entre los que se pueden mencionar la sobrepesca comercial, la captura incidental, la destrucción de hábitats críticos de alimentación, de anidación y de reposo y más recientemente la contaminación de los mares han diezmado la condición actual de las tortugas marinas (UICN 1995, Lutcavage et al. 1997). Estas razones han sido el marco causal del cual se ha originado el desarrollo de esta investigación; cuyos objetivos fueron documentar el proceso de anidación y desarrollar un diagnóstico sobre la problemática para la conservación de esta especie.

\section{MATERIALES Y MÉTODOS}

El trabajo comenzó en 1990 y se prolongó hasta 1997. Se inició en la segunda semana de febrero de cada año y se extendio hasta la última semana de julio. Playa Gandoca ubicada en el REGAMA ( $82^{\circ} 37^{\prime} \mathrm{W}, 9^{\circ} 37^{\prime} \mathrm{N}$ ) se extiende por $8.85 \mathrm{Km}$ desde Punta Mona a la esquina sureste de país (desembocadura del río Sixaola, frontera con Panamá), por su posición es una de las playas de menos acceso del país. Esta línea de costa se caracteriza por ser de alta energía asociada a una plataforma continental estrecha; prevaleciendo las corrientes marinas fuertes que se mueven en dirección norte-sur.

En general, la playa está pobremente desarrollada durante parte del año y parcialmente cubierta por basura, incluyendo cocos, troncos y una amplia variedad de desechos plásticos. La mayoría de estos desechos son originados por los centros de población, por las plantaciones de banano y los aprovechamientos forestales establecidos en la cuenca del río Sixaola.

La playa, desde Punta Mona a la boca del río Sixaola fue dividida y marcada con mojones en segmentos de 50 metros desde el norte hacia el sur con una numeración creciente, cuya finalidad fue documentar la distribución espacial de la anidación y ubicar los sitios con altas densidades de anidación para efectos de manejo del desove.

De los $8.85 \mathrm{~km}$ que playa Gandoca posee, solamente $7.4 \mathrm{~km}$ son áreas disponibles para la anidación; los $1.1 \mathrm{~km}$ restantes están en las zonas alrededor de las desembocaduras de los ríos y quebradas. Estos $7.4 \mathrm{~km}$ se dividieron en tres sectores $(A=1850 \mathrm{~m}, \mathrm{~B}=2650$ m, C $=2900 \mathrm{~m}$ ), esta segregación se desarrolló con la finalidad de ordenar los patrullajes nocturnos y facilitar el trabajo de recolección de los datos.

Todas las noches, en dos turnos (8:00 pm y 12:00 $\mathrm{mn}$ ) tres grupos de personas salieron a cada uno de los sectores en lapsos de cuatro horas, para observar y anotar todo lo pertinente a la anidación de las tortugas.

Playa Gandoca se caracteriza por aguas profundas muy cerca de la costa, ausencia de formaciones coralinas (excepto alrededor de Punta Mona), y una playa de alta pendiente que facilita el arrivo de $D$. coriacea. Las tortugas anidan justo después de la marca de marea alta o debajo de esta.

Cada hembra encontrada sin marca fue marcada después de la ovoposición con una marca metálica de MONEL. Las marcas fueron 
colocadas entre la cola y las aletas, siguiendo la metodología propuesta por Hirth y Ogren (1987).

A cada hembra encontrada se le tomaron dos medidas corporales de acuerdo con la metodología expuesta por Cornelius (1976), Pritchard et al. (1983), Hirth y Ogren (1987), Bjorndal y Carr (1989) y Guadamuz (1990). Diariamente se anotaron observaciones de la fase lunar y temperatura de la arena a una profundidad de $74.00 \mathrm{~cm}$ en el vivero (promedio de profundidad de nido para la especie). Además se verificó la hora de anidamiento, zona de la playa y número de mojón donde ocurrió la anidación, los números de las marcas en las tortugas, anotaciones sobre evidencia de marcas previas, ectobiota y daños en la piel, número de huevos normales y vanos por nido, posición de las tortuga y el nido. Se tomó como referencia el nivel de la marea, la línea de costa, profundidad del nido y posición del nido en la playa.

Los nidos fueron clasificados así: a. nido relocalizado (1. es aquel nido que fue movido de zonas de riesgo a sitios estables en la berma de la playa, 2. relocalizado en vivero), b. nido natural (el que quedó en el sitio elegido por la hembra anidadora), c. nido camuflado (nido en condición natural pero que las patrullas de trabajo cambiaron los patrones de las huellas y la fosa de anidación moldeados en la arena, con la finalidad de desorientar a los recolectores ilegales), d. nido perdido (este se dividió en 1. perdido por recolección ilegal, 2. perdido por animales domésticos, 3. perdido por erosión, 4 . perdido por depredación u otras condiciones naturales).

Todos los huevos decomisados a los recolectores ilegales por los guardias del REGAMA o los puestos en zonas de alto riesgo (100 metros alrededor de las desembocaduras de Black Creek, Middle Creek, Don Nati Creek, laguna de Gandoca y pequeñas quebradas temporales o cerca de la línea de marea baja en zonas propensas a la erosión marina) fueron relocalizados en los viveros construídos en el sector A y B.

Los viveros fueron construídos sobre la berma de la playa entre los mojones que pre- sentaron mayor anidación en años anteriores, esto con la finalidad de facilitar el manejo de los huevos y la coordinación del trabajo nocturno. Todos los viveros tuvieron un área interna promedio de 55 metros cuadrados. Los huevos colocados en los viveros fueron transplantados cuidadosamente, máximo una hora después de ser desovados u obtenidos, incluyendo los decomisados a recolectores ilegales. Los huevos fueron contados y los huevos vanos colocados en la parte superior de la nidada. Los nidos fueron rodeados con cilindros de cedazo metálico para protegerlos de la depredación por el cangrejo fantasma (Ocypode sp.). Los nidos artificiales del vivero, así como en la playa fueron relocalizados a la misma profundidad y ancho encontrados en los nidos naturales.

Lo pequeño de las submuestras de huevos para datos biométricos (15 huevos/hembras), se justifico para evitar la manipulación intensiva de cantidades superiores. En el vivero cada nido en el vivero fue monitoreado cuatro veces diariamente (0600, 1200, 1800 y 2400 horas) para determinar su temperatura como lo propuesto por Witzell y Banner (1980).

Después de la eclosión, los neonatos fueron medidos y pesados, se determinó el porcentaje de eclosión y se realizó un análisis cualitativo de los huevos no nacidos. Los neonatos se liberaron cerca del punto de avivamiento, pero nunca en el mismo lugar. El análisis de los huevos no nacidos fue realizado de acuerdo con Crastz (1982) y Whitmore y Dutton (1985).

\section{RESULTADOS}

Entre febrero de 1990 y julio de 1997, 3484 nidos fueron puestos en esta playa (en 1993 no se recolectaron datos por limitaciones financieras, Fig. 1); se estudiaron y marcaron 1 045 hembras. La mayor intensidad de anidación se dio en las áreas de mojones del 15 al 20, 31 al 36, 64 al 76 y 111 al 116 (Fig.2), siendo estos datos coincidentes con lo presentado por Chacón et al. (1996). De las 3484 nidadas, el $32 \%$ fueron puestas en la berma (Desviación Estándar, D.E.= 16.05), $37 \%$ en la zona de 


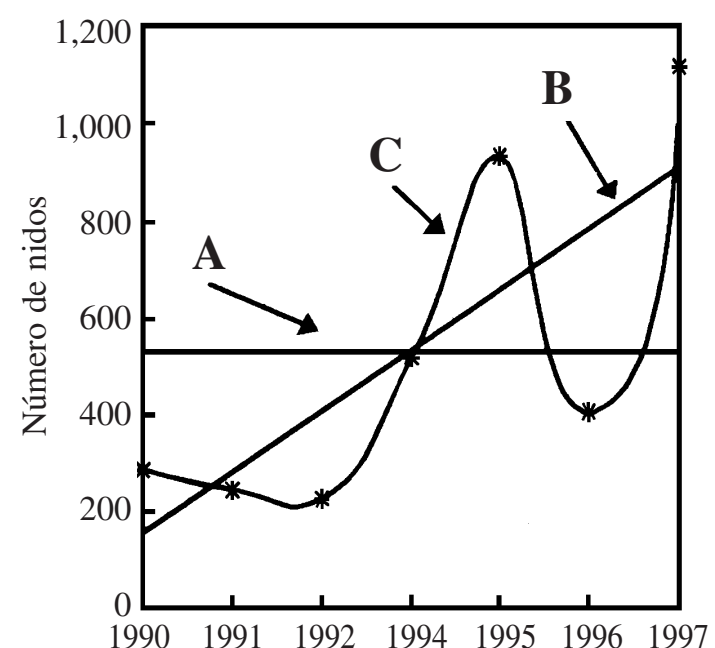

Fig. 1. Distribución de las cantidades totales de nidos por cada temporada de anidación de 1990 a 1997, A. Línea de promedio, B. Línea de mejor ajuste $\left(\mathrm{r}=0.7653, \mathrm{r}^{2}=0.5857\right.$, $a=30.5714, b=125.4286$ ), C. Curva de la distribución de los puntos. marea alta (D.E.=21.46) y $31 \%$ en la zona de marea baja (D.E.=13.10); datos similares fueron informados por Márquez (1990) y Chacón et al.(1996). Cartín (en prep.) informó que en Gandoca para 1991, el 64\% de los nidos fueron localizados entre las líneas de marea, con el subsecuente riesgo de pérdida. Los huevos puestos debajo de la cresta de la berma están ciertamente sujetos a filtraciones de agua marina de las mareas altas. Whitmore y Dutton (1985) demostraron que el desarrollo embrionario se ve adversamente afectado por las altas concentraciones de cloruro de sodio, que restringe el intercambio gaseoso y la humedad; además Leslie et al. (1996) demostraron que las infiltraciones de agua afectan el nido por el enfriamiento que provoca.

Desde 1994, se determinó que los troncos y basura traída por las corrientes marinas y la marea y depositados sobre la playa representaban una potencial barrera física que impedía a

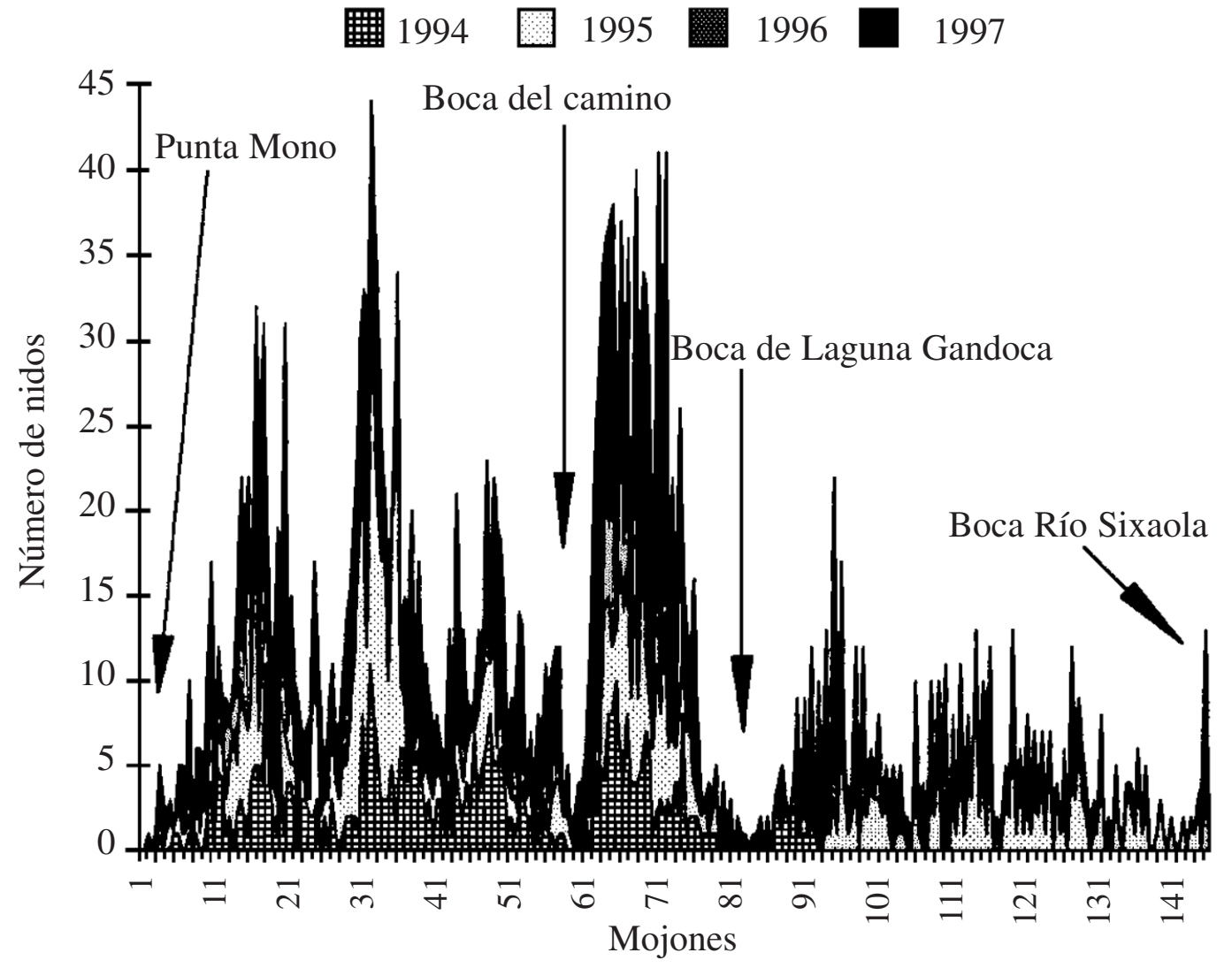

Fig. 2. Frecuencia de anidación y su distribución sobre la playa usando como puntos de referencia los mojones. 
estos reptiles alcanzar sitios más seguros para depositar sus huevos (Chacón et al. 1996); las actividades de limpieza de la playa han provocado un cambio en la tendencia observada en 1994-95 donde los porcentajes de anidación en la zona de influencia de la marea eran de $42 \%$ y $43 \%$ respectivamente, mientras que para 1996 y 1997 cuando ciertos sectores de la playa fueron limpiados estos valores descendieron a $22 \%$ y $18 \%$ respectivamente.

La presencia de 152 tortugas con marcas foráneas (University of Florida y National Marine Fisheries Service), demuestra la irregularidad en los sitios de remigración y reanidación de algunas tortugas. Estas hermbras con marcas foráneas provienen de sitios tales como: el segmento entre la desembocadura del río Matina y la desembocadura del río Pacuare en la parte norte de la costa Caribe de Costa Rica, Playa San San y playa Changuinola en Panamá; y en Playa Acandí, Golfo de Urabá, Colombia. Algunas tortugas presentaron un período de reanidamiento con un número irregular de días. Muchas tortugas visitaron playa Gandoca y retornaron una segunda vez un mes después; posiblemente en su ausencia de playa Gandoca ellas reanidaron en Panamá o en las playas de la costa norte de Costa Rica, esto se comprueba porque algunas tortugas marcadas en Gandoca han sido observadas en Mondonguillo, San San y Changuinola. Estos movimientos de anidación prueban la hipótesis del desove por disperción en una colonia de anidamiento estable según lo propuesto por Tucker (1990).

En 1997, el 8\% de las tortugas que llegaron a desovar traían marcas colocadas en 1994, $23 \%$ con marcas colocadas en 1995 y un $11 \%$ con marcas colocadas en 1996; existen datos de varias tortugas con marcas del National Marine Fisheries Service que fueron colocadas entre 1987 y 1989 (Rueda et al. 1992). Estos datos manifiestan que la colonia anidadora de Gandoca está constituída por remigrantes con un ciclo de anidamiento de 3, 2, 1 años respectivamente; aunque la tendencia demuestra un patrón predominante de reanidación de cada dos años, hecho coincidente con Hughes (1996).
La hipótesis de que en el Mar Caribe existe una megapoblación de tortugas baulas conformada por colonias anidadoras es importante como justificación para usar los estudios y comparaciones de las diferentes colonias como un punto de partida en busca de describir y entender esta megapoblación (Tucker 1990)

Las series de la marcas utilizadas en estas temporadas fueron D7100-D7400, 57801-57900, 61001-61990, 78001-78150, D9001-D9099, D9100-D9680 (University of Florida), las tortugas baulas que mostraron reanidación de dos o más veces variaron su lapso de visita en un ámbito de 8 a 12 días, con una moda estadística de 9 días; esto es coincidente con los datos presentados por National Research Council (1990), Boulon et al. (1996), Steyermark et al. (1996) y Miller (1997).

Un análisis comparativo de datos para playa Gandoca, expuso que los más importantes meses para la anidación son abril $(38 \%)$ y mayo $(33 \%)$ de acuerdo con Chacón et al. (1996), Leslie et al. (1996), Campbell, Lagueux y Mortimer (1996), (Fig. 3); esta información además es coincidente con Pritchard (1971) y Márquez (1990). El promedio total anual de nidos en playa Gandoca fue de 534 con una densidad de 7.22 nidos/100 $\mathrm{m}$ de playa, un promedio de 4 tortugas por noche por temporada, aunque el máximo de hembras anidadoras por noche fue de 42 , mientras que el mínimo fue de cero.

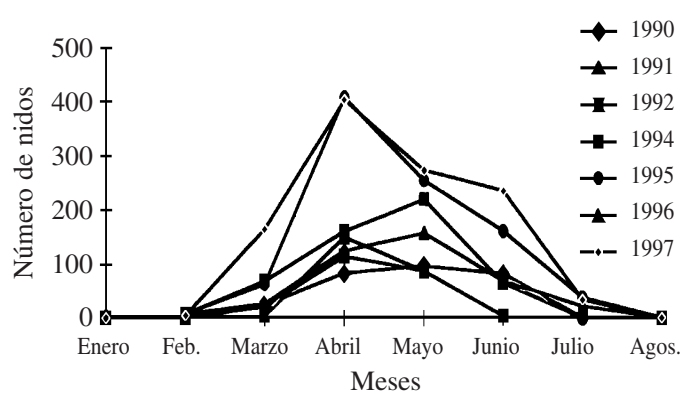

Fig. 3. Frecuencia de anidación mensual para las temporadas de 1990 a 1997. 
El 54\% de las tortugas emergieron durante las fases oscuras de la luna, a pesar de que los análisis estadísticos no mostraron una significancia contundente (t-student, $\mathrm{p}>0.5$ ). La base teórica soporta la hipótesis de que el comportamiento selectivo por el cual las tortugas visitan la playa en noches oscuras es resultado de una estrategia para sobrevivencia al disminuir el riesgo de depredación.

El promedio de la longitud curva del caparazón de 1045 tortugas muestreadas fue 154.65 cm (D.E.= 2.1213), (Cuadro 1, Fig. 4), el cual está dentro de los ámbitos expuestos por otros investigadores en Gandoca y otros sitios (Hirth y Ogren 1987, Chu 1990, Hall 1990, McDonald et al. 1993, Chacón et al. 1996). El promedio del ancho curvo fue de $112.83 \mathrm{~cm}$ (D.E.= 5.6569), las clases para la longitud curva del caparazón más importantes están representadas entre $145.1-150.0 \mathrm{~cm}(21.1 \%), 150.1-155.0 \mathrm{~cm}$ (23.72\%) y $155.1-160.0 \mathrm{~cm}(23.51 \%$; la mínima longitud curva encontrada fue de $105.0 \mathrm{~cm}$, mientras que la máxima longitud curva del caparazón fue de $205.0 \mathrm{~cm}$.

En una muestra de 2045 nidos el número promedio de huevos por nido fue de 114 hue-

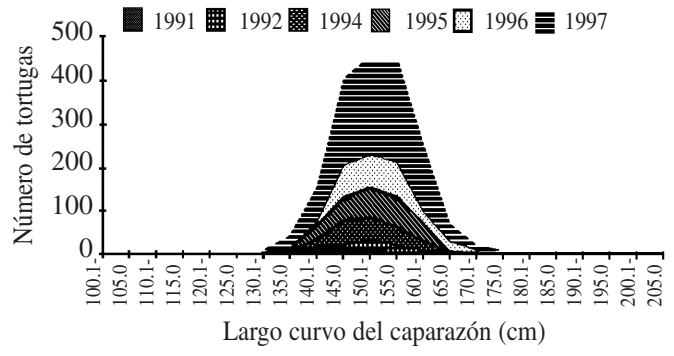

Fig. 4. Clases de longitud curva del caparazón para las hembras anidadoras en playa Gandoca, 1991-1997.

vos. El promedio de huevos normales fue 79.28 (D.E.= 2.7740, Cuadro 2). 1250 huevos normales fueron seleccionados al azar de 80 hembras anidadoras para ser medidos; el diámetro promedio fue de $52.1 \mathrm{~mm}$ (D.E.: 3.1301). El diámetro promedio de los huevos de algunas colonias de baulas varió entre 50 y 55 mm (Hirth y Ogren 1987; Campbell, Lagueux y Mortimer 1996, Leslie et al. 1996), mientras que Miller (1997) informó de un promedio de $53.4 \mathrm{~mm}$ (D.E.=0.5) para el diámetro de los huevos de 9 colonias de anidación diferentes. En Tortuguero, Costa Rica; Leslie et al. (1996) encontraron que el diámetro para

\section{CUADRO 1}

Comparación del promedio de la longitud curva del caparazón de las hembras que desovan en Gandoca con las hembras de otras colonias de anidación

\begin{tabular}{|c|c|c|c|c|c|}
\hline $\begin{array}{l}\text { Playa y/o } \\
\text { País }\end{array}$ & $\begin{array}{l}\text { Año } \\
\text { estudiado }\end{array}$ & Referencia & LCC & Ambito & D.E \\
\hline Gandoca & 1991 & Cartín (en prep.) & 153.5 & $134-166$ & n.i \\
\hline Gandoca & 1992 & Quirós (en prep.) & 153.3 & $122-168$ & 7.7 \\
\hline Gandoca & 1994 & Chacón et al. (1996) & 153.8 & $115-205$ & 10.4 \\
\hline Gandoca & 1995 & Esta publicación & 154.05 & $126-183$ & 8.6 \\
\hline Gandoca & 1996 & Esta publicación & 156 & $105-205$ & 7.82 \\
\hline Gandoca & 1997 & Esta publicación & 154.7 & $187-112$ & 2.21 \\
\hline Mondonguillo & 1994 & Chaves (en prep.) & 154.01 & $133-179$ & 7.32 \\
\hline Parismina & 1986 & Hirth \& Ogren (1987) & 152.8 & $134-171$ & n.i. \\
\hline Tortuguero & $1990-91$ & Leslie et al. (1996) & 156.2 & $124-180.3$ & 10.6 \\
\hline Tortuguero & 1995 & Campbell , Lagueux \& Mortimer (1996) & 152.8 & $138-179.1$ & 8.9 \\
\hline Tortuguero & 1996 & Lahanas \& Kerr (en prep.) & 153.2 & $133-168$ & 7.22 \\
\hline Puerto Rico & 1990 & Hall (1990) & 155.3 & $140-168$ & 6.6 \\
\hline Puerto Rico & 1991 & Tucker \& Frazer (1991) & 155 & n.i. & n.i. \\
\hline Papua & 1989 & Hirth (1993) & 161.35 & $145-180$ & 8.77 \\
\hline Sand Croix & 1993 & McDonald et al. (1993) & 152.8 & $131-177$ & n.i. \\
\hline
\end{tabular}

LCC: Promedio de Longitud Curva del Caparazón en cm. n.i.: No hay información 


\section{CUADRO 2}

Valores de la cantidad promedio de huevos normales por nido para las temporadas de anidación de 1994 a 1997 en playa Gandoca y su comparación con otras colonias de anidación.

$\begin{array}{llllll}\begin{array}{l}\text { Playa y/o } \\ \text { País }\end{array} & \begin{array}{l}\text { Año } \\ \text { estudiado }\end{array} & \text { Referencia } & \text { LCC } & \text { Ambito } & \text { D.E } \\ \text { Gandoca } & 1994 & \text { Chacón } \text { et al. } \text { (1996) } & 79.4 & 5-158 & 20.62 \\ \text { Gandoca } & 1995 & \text { Esta publicación } & 79.4 & 1-156 & 17.78 \\ \text { Gandoca } & 1996 & \text { Esta publicación } & 85 & \text { n.i. } & 16.28 \\ \text { Gandoca } & 1997 & \text { Esta publicación } & 81.8 & 9-160 & 17.02 \\ \text { Tortuguero } & 1990 & \text { Leslie } \text { et al. }(1996) & 86 & 77-115 & \text { n.i. } \\ \text { Tortuguero } & 1991 & \text { Leslie } \text { et al. } \text { (1996) } & 80 & 58-114 & \text { n.i. } \\ \text { Tortuguero } & 1995 & \text { Campbell, Lagueux \& Mortimer (1996) } 80.2 & 48-117 & 27.39 \\ \text { Tortuguero } & 1996 & \text { Lahanas \& Kerr (en prep.) } & 86.9 & 20-167 & 17.6 \\ \text { 12 } & 1997 & \text { Miller } \text { (1997) } & 81.5 & \text { n.i. } & 3.6 \\ \text { Puerto Rico } & 1984-1987 & \text { Tucker \& Frazer (1994) } & 74.88 & 24-120 & \text { n.i. } \\ \text { Sand Croix } & 1981-1995 & \text { Boulon } \text { et al. } \text { (1996) } & 79.7 & 72.9-85.9 & 3.99\end{array}$

N.H.: Promedio de huevos normales por nido.

12: Número de poblaciones incluídas en este dato.

n.i.: No hay información

huevos de las temporadas en 1990 y 1991 fue de $54.0 \mathrm{~mm}$ (D.E.=1.4, ámbito 47.5-58.0 mm).

La variación de la cantidad de huevos normales desovados por tortugas (a las cuales se les dio seguimiento constante por al menos 6 desoves durante la misma temporada), fue irregular. Es decir no se evidenció una tendencia concluyente acerca del metabolismo de la baula y su relación con las cantidades de huevos desovados.

La tortuga baula desova un número de pequeños huevos sin vitelo denominados "vanos". En una muestra de 2045 huevos, el porcentaje promedio de huevos vanos por nido fue de 30.7\% (35 huevos/nidos, D.E.=11.3), en el total de nidadas. Chacón et al. (1996) informaron un $31.5 \%$ de huevos vanos por nidada, mientras Campbell, Lagueux y Mortimer (1996) encontraron un promedio de 28.4 huevos vanos/nido. En una muestra de 40 nidos, el diámetro de los huevos vanos ociló entre 4-48 $\mathrm{mm}$ y la moda estadística fue de $30 \mathrm{~mm}$. La mayoría de los huevos vanos fueron desovados al finalizar la ovoposición. Tres posibles valores adaptativos tienen los huevos vanos: distracción a depredadores, amortiguamiento termal y prevención de derrumbes de arena dentro de los huevos normales, asi permitiendo el intercambio gaseoso y previendo la deformación de embriones por el apermasamiento de las posturas (Hall 1990).

Entre 1990 y 1997, un total de 726 nidos con 58080 huevos normales fueron colocados en los viveros. El período promedio de incubación en el vivero fue de 61.00 días (D.E. $=8.50$, ámbito: 47-72 días).

La tasa promedio de avivamiento en vivero fue de $46.14 \%$ (D.E.= 25.14, ámbito 0$96 \%$ ), lo que representó la liberación de 26,798 neonatos; en Malasia varió de 32 a $71.5 \%$ en un período de diecinueve años (Siow y Moll 1982, Mortimer 1990). Whitmore y Dutton (1985) informaron un $68.7 \%$ de sobrevivencia en nidadas reubicadas de 50 huevos cada una en Surinam. Chacón et al. (1996) informa de $42 \%, 15 \%$ y $54.4 \%$ de sobrevivencia en viveros para playa Gandoca respectivamente y Alvarado y Figueroa (1989) informa de $69.9 \%$ de sobrevivencia para viveros de huevos de baulas en México.

De los viveros se tomaron 237 neonatos, fueron medidos y pesados unos pocos minutos después de haber emergido. El promedio de la longitud linear estándar del caparazón fue de $59.61 \mathrm{~mm}($ D.E.= 4.26) y el promedio del peso total fue de $44.38 \mathrm{~g}$ (D.E.= 6.12). Hirth $\mathrm{y}$ 
Ogren (1987) informaron que el promedio de longitud de caparazón de neonatos para colonias de baulas varió entre 55 y $63 \mathrm{~mm}$ y el promedio del peso estuvo entre 39 y $47 \mathrm{~g}$. Los pesos y longitudes son coincidentes con los datos presentados por Miller (1997).

Los nidos fueron exhumados una semana después del primer nacimiento y los huevos no nacidos fueron abiertos y fue anotado su contenido según lo siguiente: desechos de huevos cuya condición los hace "indescifrables" (10.09\%); ninguna señal visible de un embrión (32.15\%); pre inicio de embrión (21.96\%); embrión totalmente formado $(21.5 \%)$, neonatos muertos en la cavidad de ascenso del nido (14.3\%).

Los valores de los nidos de control $(n=418)$ dejados en estado natural y monitoreados semanalmente mostraron que el porcentaje de avivamiento promedio fue $39 \%$ con un ámbito de 0\% a 65\% y una D.E.= 25.7803; lo que demuestra (a diferencia de otras playas) que la eclosión natural en Gandoca es baja, comparada con los datos expuestos por Hirth y Ogren (1987) y Guadamuz (1990); justificando claramente la necesidad de desarrollar actividades que fortalezcan la sobrevivencia de la especie.

\section{DISCUSIÓN}

La mayoría de las tortugas son animales longevos que tardan en alcanzar su madurez sexual y que poseen tasas de sobrevivencia altas cuando ya son adultos (UICN 1995). Pero en este ciclo de vida tan peculiar, existen etapas que son muy sensibles; entre ellas está la fase de los huevos. Siendo este estado la base de toda su pirámide poblacional, los impactos sobre ellos crean cambios en las colonias de anidación a largo plazo (Spotila et al. 1996). La recolección excesiva de huevos o la cacería de las hembras anidadoras tendrá efectos tangibles inmediatos en la disminución de nidos y arribo de hembras anidadoras hasta algunas décadas en el futuro. Entre tanto no hay señales visibles de un declive de la población, lo que provoca que las comunidades y el público en general se enteren de sus impactos hasta cuando el colapso es un hecho irreversible. Betz y Welch (1992) presentan ejemplos de ello como los siguientes números para Irian Java, Indonesia: 11000 nidos (1956), 6721 nidos (1968), 280 nidos (1990). Para los mismos autores el $97 \%$ de las causas de este colapso se deben a la recolección de los huevos. Spotila et al. (1996), han estimado la población mundial entre 26 000 y 42900 tortugas baulas (promedio estimdo 34,500). Establecieron además que con la tendencia actual en 18 años la población mundial habra sido extinguida de la Tierra. Otras observaciones recolectadas como la ectobiota y daños en la piel no mostraron patrones claros que pudieran ser ampliamente discutidos.

Durante el estudio los más importantes inconvenientes fueron: la recolección ilegal de huevos (Fig. 5), siendo una problemática compartida con otras playas (Campbell, Lagueux y Mortimer 1996, Leslie et al. 1996), la depredación por animales domésticos (1\% del total de los nidos anuales) y la erosión oceánica (7\% del total de los nidos anuales); esos impactos llevaron a la desición de construir viveros como una estratégia de conservación.

Solamente los desoves localizados cerca de la desembocadura de las quebradas, en las zonas intermareales, los obtenidos en período de alta visitación humana o los desoves decomisados a los recolectores ilegales, fueron colocados en el vivero. Se asumió que la tasa de

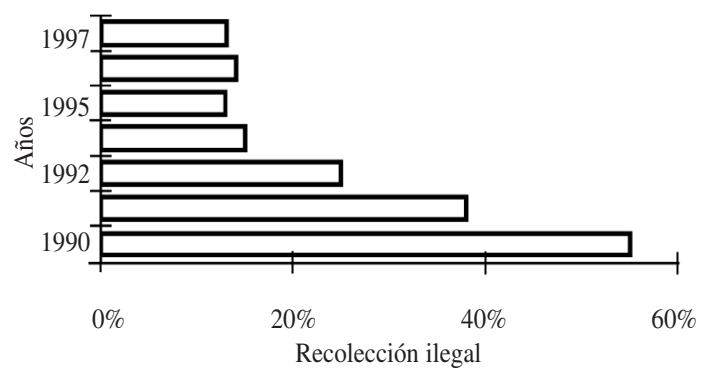

Fig. 5. Frecuencia porcentual para la recolección ilegal de huevos en playa Gandoca de 1990 a 1997. 
avivamiento para esos huevos probablemente era de $0 \%$ si se quedaban en la playa debido a que eran proclives a ser impactados.

Las patrullas de campo informaron ver los primeros avivamientos en la tercera semana de mayo en los nidos naturales de control, los nidos naturales camuflados y los nidos relocalizados, siendo mismo periodo durante el cual los neonatos empezaron a nacer en los viveros.

En playa Gandoca existen períodos de baja temperatura y alta precipitación; la temporada de anidación se vio afectada por estos cambios climáticos que influyen directamente sobre la temperatura de incubación y la proporción de sexos en cada avivamiento. Del análisis de los valores de la temperatura, se desprende que la temperatura de incubación varió alrededor de la temperatura pivotal $\left(29.5^{\circ} \mathrm{C}\right)$, cada mes; para meses como abril y las dos primeras semanas de marzo la temperatura de incubación se mantuvo a niveles por los cuales se producen machos en los neonatos; mientras que en junio y julio la temperatura de incubación fue más alta que la pivotal tendencia que provoca la producción de hembras en los neonatos.

Este patrón de temperaturas de incubación variantes alrededor de la temperatura pivotal también ha sido encontrado en otras playas del Caribe de Costa Rica, siendo documentado ampliamente por Leslie et al. (1996) en playa Tortuguero.

Una evaluación ecológica rápida expuso que los nidos tienen un alto riesgo de perderse en los $100 \mathrm{~m}$ alrededor de la desembocadura de las quebradas y también en los segmentos de playa suceptibles a erosión marina (entre los mojones 6-11, 40-61, 67-73 y 80-95) y segmentos en los cuales existe fácil acceso a los recolectores ilegales (mojones 64 hacia el sur y hasta el 145).

Los problemas ambientales más graves que afectaron la colonia anidadora de baulas en Playa Gandoca fueron:

1. Depredación por recolectores ilegales comenzó a ser un serio impacto cuando esta recolección superó el número de nidos salvados por noche,
2. Destrucción o modificación de la playa de anidación como efecto de la deforestación en el continente que causa severos problemas en la playa cuando en la época lluviosa el oceáno acarrea a ésta troncos y otros desechos. En el oceáno y la corriente litoral deposita la basura vegetal sobre la playa, cambiando su morfología y bloqueando el acceso a las tortugas. El sector más afectado está en las cercanias de la desembocadura del río Sixaola. La madera y otra clase de basura vegetal estuvo acompañada por plásticos, metales y otros tipos de basura agrícola y doméstica,

3. Depredación por animales domésticos. Algunos recolectores ilegales enseñan a perros a buscar las nidadas y cuando las encuentran reciben algunos huevos como "premio". Los perros subsecuentemente escarban y comen el contenido de cada nido incluyendo neonatos. Los cerdos también son depredadores activos de huevos y crías. Los caballos y vacas compactan la arena, colapsando nidos, apermasando la arena y como consecuencia de esto los neonatos mueren,

4. Otros factores causados por el hombre: a. la explotación de la arena como material para reparar el camino a Gandoca, b. el drenaje temporal (inducido) de las quebradas y pantanos, c. la escorrentía y descarga agrícola, d. la luz artificial de linternas y fogatas, e. los vehículos sobre la playa, f. los productos plásticos y pesticidas de las plantaciones bananeras que llegan a la playa por los ríos.

Como soluciones a estos impactos se presenta la creación de mecanismos adecuados para: 1. la clausura del camino antes de llegar a la playa y las actividades de control especialmente en los períodos de la mayor visitación de turismo a playa Gandoca, 2. diseñar una estrategia cogestionaria comunidad-proyecto para aplacar el mercado negro, que comercia con carne, huevos y escamas de concha de tortuga, 3. realizar estudios binacionales, cooperación interproyectos y retroalimetación entre los ac- 
tores claves de América Central, 4. el manejo y protección de los principales segmentos de anidación en la playa, 5. el desarrollo de material educacional y talleres de educación ambiental para la gente local (en Gandoca y las comunidades de la zona de amortiguamiento), 6. el manejo y protección de todos los estadios de vida de la baula (huevos, neonatos y adultos), 7. desarrollar un estudio socioeconómico de la gente local para incluirlos en el programa de uso doméstico de huevos de baula y buscar alternativas de subsistencia para los recolectores ilegales y la gente local.

Todas estas actividades de conservación son necesarias para futuras temporadas de anidación.

\section{AGRADECIMIENTOS}

El financiamiento fue provisto principalmente por Frankfurt Zoological Society (proyecto 1219/97), PROARCA-Costas (The Nature Conservancy, World Wildlife Fund, University of Rhode Island), el Corredor Biológico Talamanca-Caribe, General Service Foundation y Tropica Verde. Mi agradecimiento a los jerarcas y administración del Refugio Nacional de Vida Silvestre Gandoca/Manzanillo. También expreso el más profundo apoyo y respeto a la comunidad de Gandoca y a todos los voluntarios quienes dieron al proyecto alta calidad en la ayuda de campo; sin ellos el trabajo de investigación hubiera sido imposible.

También doy gracias a la Corporación para la Conservación Caribeña, y a nuestros colegas de ANAI en San José y Talamanca. Este proyecto fue realizado como parte del Programa Marino de ANAI.

\section{RESUMEN}

La anidación de la tortuga baula fue estudiada en playa Gandoca, una importante playa de anidación de tortugas marinas localizada al sureste de la costa Caribeña de Costa Rica, dentro de los límites del Refugio Nacional de Vida Silvestre Gandoca/Manzanillo (82 $37^{\prime} \mathrm{W}, 09^{\circ} 37^{\prime}$ N). Entre 1990 y 1997, se desarrollaron campañas de observación de la anidación entre los meses de febrero a ju- lio de cada año; 16 variables de la anidación fueron anotadas y una parte de la colonia fue marcada con marcas monel y 3484 nidos fueron encontrados; durante este periodo se depositaron un promedio de 534 nidos por temporada, 1,135 nidos fue el máximo de anidación anual, mientras que 226 nidos fue el mínimo, el $20.8 \%$ de los nidos fueron relocalizados en los viveros. El promedio de la longitud curva del caparazón de las hembras fue de $154.65 \mathrm{~cm}$ y el promedio del ancho curvo del caparazón fue de $112.83 \mathrm{~cm}$. Las observaciones mostraron que el promedio de huevos normales para este periodo fue de 79.28, mientras que el promedio de huevos vanos fue de 35 . La recolección ilegal de huevos, la basura sobre la playa y la erosión intensiva son parte de los problemas más importantes que están causando el declive de las tortugas en Gandoca.

\section{REFERENCIAS}

Alvarado, J. \& A. Figueroa. 1989. Ecología y Conservación de las Tortugas Marinas de Michoacán, México. Universidad Michoacana de San Nicolas de Hidalgo. Cuadernos de Investigación 7. 72 p.

Betz, W. \& M. Welch. 1992. La que fuera una floreciente colonia de tortugas de cuero en Irian Java, Indonesia, está declinando. Noticiero de Tortugas Marinas (WIDECAST) 56: 8-9.

Bjorndal, K. \& A. Carr. 1989. Variation in clutch size and eggs size in the green turtle nesting population at Tortuguero, Costa Rica. Herpetology 45: 181-189.

Boulon, R., P. Dutton \& D. McDonald. 1996. Leatherback Turtles (Dermochelys coriacea) on St. Croix, U.S. Virgin Island: Fifteen Years of Conservation. Chelonian Cons. Biol. 2: 141-147.

Campbell, C., C. Lagueux \& J. Mortimer. 1996. Leatherback Turtle, Dermochelys coriacea, Nesting at Tortuguero, Costa Rica, in 1995. Chelonian Cons. Biol. 2: 168-172.

Carr, A., M. Carr \& A. Meylan. 1990. Ecología y Migración de las tortugas marinas, 7. Colonia de tortuga verde en el Caribe Occidental. Florida Marine Research Institute \& Caribbean Conservation Corporation. Gainesville, Florida. 42 p.

Chacón, D., W. McLarney, C. Ampie \& B. Venegas. 1996. Reproduction and conservation of the leatherback sea turtle Dermochelys coriacea (Testudines: Dermochelyidae) on Gandoca, Costa Rica. Rev. Biol. Trop. 44: 853-860.

Chu, L. 1990. Observations on the nesting population of leatherback turtles Dermochelys coriacea in Trinidad. Caribb. Mar. Stud. 1:48-53. 
Cornelius, A., M. Ulloa, J. Castro, M. Mata \& D. Robinson. 1986. Management of Olive Ridley Sea Turtles (Lepidochelys olivacea) nesting at playa Nancite and Ostional, Costa Rica. In: Robinson G.J. \& K.H. Redford. Neotropical Wildlife Use and Conservation. University of Chicago, Chicago, Illinois. 111-135 p.

Cornelius, S. 1976. Marine Turtles nesting activity at Playa Naranjo, Costa Rica. Brenesia 8:1-27.

Crastz, F. 1982. Embryological stages of the marine turtle Lepidochelys olivacea (Eschscholtz). Rev. Biol. Trop. 30:113-120.

Guadamuz, N. 1990. Registro de Anidamiento de Dermochelys coriacea (tortuga Baula) en Playa Grande de Matapalo, Santa Cruz-Guanacaste. Escuela de Ciencias Biológicas, Universidad Nacional, Heredia, Costa Rica. 60 p.

Hall, K. 1990. Hatchling success of leatherback turtle (Dermochelys coriacea) clutches in relation to biotic and abiotic factors. In: Richardson T.H., H.I. Richardson \& M. Donnelly. Proc. of the Tenth Annual Workshop on Sea Turtle Biology and Conservation. NOAA. Technical Memorandum NMFS-SEFC-278. $286 \mathrm{p}$.

Herzog, P. \& M. Gerrand. 1992. An Assessment of Ecoturism and its impact on Leatherback Sea Turtles at Playa Grande, Costa Rica. Programa Regional en Manejo de Vida Silvestre, Universidad Nacional. Heredia, Costa Rica. 44 p.

Hirth, H. \& L. Ogren. 1987. Some Aspects of the Ecology of the Leatherback Turtle Dermochelys coriacea at Laguna Jalova, Costa Rica. NOAA Technical Report NMFS 56. 14 pp.

Hughes, G. 1996. Nesting of the Leatherback Turtle (Dermochelys coriacea) in Tongaland, KwaZulu-Natal, South Africa, 1963-1995. Chelonian Cons. Biol. 2: 153-158.

Leslie, A., D. Penick, J. Spotila \& F. Paladino. 1996. Leatherback Turtle, Dermochelys coriacea, Nesting and Nest Success at Tortuguero, Costa Rica, in 19901991. Chelonian Cons. Biol. 2: 159-168.

Lutcavage, M., P. Plotkin, B. Witherington \& P. Lutz. 1997. Human Impacts on Sea Turtle Survival. In: Lutz, P. \& J. Musick (eds). The Biology of Sea Turtles. CRC, Boca Ratón, Florida. p. 387-404.

Márquez, R. 1990. FAO Species catalogue. Vol 11: Sea Turtles of the world. An annotated and illustrated catalogue of sea turtle species known to date. FAO Fisheries Synopsis, FAO, Roma. $\mathrm{N}^{\circ} 125.81 \mathrm{p}$.
McDonald, D., P. Dutton \& R. Boulon. 1993. Tagging and Nesting Research on Leatherback Sea Turtles (Dermochelys coriacea) on Sandy Point, St. Croix, U.S. Virgin Islands. U.S. Fish and Wildlife Service, Dept. Planning and Natural Resources. 26 p.

Meylan A., A. Bass, D. Crouse, M. Donnelly, K. Eckert \& R. Márquez. 1997. Biology and Status of the Hawkbill in the Caribbean. IUCN/SSC Marine Turtle Specialist Group, Washington, D.C. 53 p.

Miller, J. 1997. Reproduction in Sea Turtles. In: Lutz, P. \& J. Musick(eds), p. 52-81. In The Biology of Sea Turtles. CRC, Boca Ratón, Florida.

Mortimer, J. 1990. Marine Turtle Conservation in Malaysia. In T.H. Richardson, H.I. Richardson \& M. Donnelly (eds.). Proc. of the Tenth Annual Workshop on Sea Turtle Biology and Conservation. NOAA. Technical Memorandum NMFS-SEFC-278. 286 p.

National Research Council. 1990. Decline of the sea turtles: Causes and Prevention. National Academy, Washington, D.C. 259 p.

Pritchard, P. 1971. The leatherback or leathery turtle Dermochelys coriacea. IUCN. Monogr. No. 1. 39 p.

Pritchard, P., P. Bacon, F. Berry, A. Carr, J. Fletmeyer, R. Gallagher, S. Mopikins, R. Lankford, R. Marquez, L. Ogren, W. Pringle, H. Richard \& R. Whitan. 1983. Manual sobre tecnicas de investigación y conservación de las tortugas marinas. Center for Environmental Education. Washington, D.C. 133 p.

Rueda, J.V.; G.A. Ulloa \& S.A. Medrano. 1992. Estudio sobre la Biología reproductiva, la ecología y el manejo de la tortuga canal (Dermochelys coriacea) en el Golfo de Urabá. Inst. Nac. de los Recursos Nat. Renov. y del Amb. (INDERENA), Bogotá, Colombia, p. 62-65.

Siow, K. T. \& E. O. Moll. 1982. Status and conservation of estuarine and sea turtles in West Malaysian waters. In Bjorndal, K. A. (ed.). Biology and conservation of sea turtles. Smithsoniam Institute. Washington. D.C. 339-347 p.

Spotila, J., A. Dunhan, A. Leslie, A. Steyermark, P. Plotkin \& F. Paladino. 1996. Worldwide Population Decline of Dermochelys coriacea: Are Leatherback Turtles Going Extinct?. Chelonian Cons. Biol. 2: 209-222.

Spotila, J., A. Leslie \& F. Paladino. 1996. Population cycles or Population Decline: Are the Leatherback Turtles going extinc?. In A. Keinath et. al. Proc. of the Fifteenth Annual Workshop on Sea Turtle Biology and Conservation. NOAA. Technical Memorandum, NMFS-SEFC-387. 308 p. 
Steyermark, A., K. Williams, J. Spotila, F. Paladino, D. Rostal, S. Morreale, M. Koberg \& R. Arauz. 1996. Nesting Leatherback Turtles at Las Baulas National Park, Costa Rica. Chelonian Cons. Biol. 2: 173-183.

Tucker, A. 1990. A test of the scatter-nesting hypothesis at a seasonally stable leatherback rookery. Richardson, T.H.; H.I. Richardson \& M. Donnelly (compilers). Proc. of the Tenth Annual Workshop on Sea Turtle Biology and Conservation. NOAA. Technical Memorandum NMFS-SEFC-278. 286 p.

Tucker, A \& N. Frazer. 1994. Seasonal variation in Clutch Size of the Turtle, Dermochelys coriacea. J. Herpetol. 28: 102-109.

Unión Internacional para la Conservación de la Naturaleza y de los Recursos Naturales. 1995. Estrategia Mundial para la Conservación de las Tortugas Marinas. Grupo de Especialistas de Tortugas Marinas UICN/CSE. Arlinton, Virginia. 24 p.

Whitmore, P. \& P. Dutton. 1985. Infertility embryonic mortality and nest site selection in leatherback and green sea turtles in Suriname. Biol. Conserv. 34: 251-272.

Williams, K. A. Steyermark, C. Hurd, A. Schwandt, F. Paladino \& J. Spotila. 1995. Population Ecology, nesting and Success of leatherback turtles, Dermochelys coriacea, at Las Baulas Guanacaste National Park, Costa Rica. In Keinarth, J., D. Barnard, J. Musick \& B. Bell. Proc. of the fifteenth Ann. Symposium on Sea Turtle Biology and Conservation. NOAA. Technical Memorandum. NMFS-SEFSC387. 355 p.

Witzell, W. \& A. Banner. 1980. The hawksbill turtle (Eretmochelys imbricata) in western Samoa. Bull. Mar. Sci. 30: 571-579

Yesaki, M. \& M. Giudicelli. 1971. Summary of exploratory Fishing operations of the R/V Canopus in the Caribbean Sea from December 1968 to June 1970. Regional Project of the fisheries development in Central America. CCDP-FAO-PNUD, Roma. 28 p. 